\title{
Graduate certificate programs provide both motivation and flexibility for careers in bioinformatics and biomedicine: experience of George Mason University, Virginia, USA
}

\author{
A. Baranova, V. Chandhoke \\ School of Systems Biology, George Mason University, Fairfax, VA, United States \\ *e-mail:aancha@gmail.com
}

Key words: graduate certificates, non-degree programs, bioinformatics, laboratory and data management, career development

Motivation and Aim: The mission of the School of Systems Biology (SSB) at George Mason University (GMU) is to provide diverse population of post-baccalaureate and graduate students with a research and educational environment that allows integration of the various areas of molecular and cellular biosciences from a systems perspective. To this end, the SSB offers research-based degree programs whose graduates go on to pursue careers in academy, government agencies, private industry, higher education, and health care.

Since mid-2000, SSB GMU offers MS and PhD programs in Biology/Biosciences as well as in Bioinformatics and Computational Biology. In all four of these programs together, current headcounts reached more than 150 students, with 40 students graduating each academic year. Fifteen years long experience with managing these programs led us to understanding that "traditional" MS (30-32 credits) and PhD (72 credits) programs do not always fit the goals of post-baccalaureate students.

Results: To this end, two specialized non-degree Certificate programs were developed: 1) Graduate Certificate in Personalized Medicine (15 credits); 2) Graduate Certificate in Bioinformatics and Computational Biology (15 credits). In each program, twelve out of 15 credits may be transferred to any "traditional" MS or PhD program offered by SSB. As an example, the following groups are identified as target groups for enrolling in Certificate Program in Personalized Medicine:

1) Non-Physician Healthcare professionals currently employed as healthcare administrators, physician assistants, nurses and healthcare technicians, nutritionists, dieticians, etc;

2) Recent BS or BA graduates seeking admission to medical graduate programs (pre-med and pre-dental students);

3) Healthcare IT professionals, computational biologists and bioinformaticians with attained degrees;

4) R\&D professional in the Drugs and Diagnostics fields;

5) Sales and other professional in biomedical and insurance industry;

6) Bench scientists in different branches of biology (predominantly at BS and MS levels);

7) Health industry economists, Government policy makers and regulators, Patient advocates and media experts;

8) Employees and associates of biomedicine-related investment funds.

Both programs were successful in collecting substantial amount of applications: Certificate in Personalized Medicine $(N=14)$ was more attractive than Certificate in Bioinformatics and Computational Biology $(N=2)$. In 2017, Certificate was then re-evaluated and expanded into Professional (non-Thesis) Masters in Bioinformatics 
Management, also made available as a hybrid online degree. Superior attractiveness of Certificate in Personalized Medicine for potential applicants is explained by initial lack of advertising and the fact that an inception of the programs, only a few educational institutions offered training in Personalized Medicine. Given that this program responds to a need in educated workforce that is able to understand, interpret and implement recent discoveries in genomics, proteomics, and metabolomics in both basic and clinical research settings, it provides its graduates a competitive edge over BS in Biology alumni when they apply for professional jobs, even in absence of graduate degree.

By interviewing the students accepted to the Certificate in Personalized Medicine, we found that an overwhelming majority of these students see the Certificate program as a stepping stone toward the Master's or $\mathrm{PhD}$ degree, which they typically intend to complete within the same University.

Conclusion: Graduate certificate programs provide both motivation and flexibility for careers in bioinformatics and biomedicine, and facilitate educational access for professional populations of students by providing step-wise re-immersion into academic environment.

Acknowledgements: Supported by the College of Science, GMU, Virginia, USA. 